Published in final edited form as:

Clin Neuropharmacol. 2014 ; 37(3): 69-72. doi:10.1097/WNF.0000000000000033.

\title{
A Trial of D-Cycloserine to Treat Stereotypies in Older Adolescents and Young Adults With Autism Spectrum Disorder
}

\author{
Maria Urbano, MD, Leonore Okwara, MPH${ }^{\star}$, Paul Manser, $\mathbf{M S}^{\dagger}$, Kathrin Hartmann, $\mathrm{PhD}^{\star}$, \\ Amy Herndon, BS ${ }^{*}$, and Stephen I. Deutsch, MD, PhD* \\ "Department of Psychiatry and Behavioral Sciences, Eastern Virginia Medical School, Norfolk \\ †Department of Biostatistics, Virginia Commonwealth University, Richmond, Virginia.
}

\begin{abstract}
Objectives-Autism spectrum disorders (ASDs) have core impairments in social communication as well as the presence of repetitive, stereotypic behaviors and restricted interests. Older adolescents and young adults are particularly impacted by these deficits. Preclinical data implicate glutamatergic dysfunction in the pathophysiology of ASDs. D-Cycloserine (DCS), a partial glycine $_{\mathrm{B}}$ agonist at the N-methyl-D-aspartic acid receptor site, has been shown to improve sociability in mouse models and a small human study. The sensitivity of the obligatory glycine ${ }_{B}$ co-agonist binding site may change with daily administration of DCS as a result of agonistinduced desensitization. The efficacy of a "pulsed" once-weekly administration versus "daily" administration of DCS was compared.
\end{abstract}

Methods-Males and females, ages 14 to 25 years, with a Diagnostic and Statistical Manual of Mental Disorders, Fourth Edition Text Revision diagnosis of an ASD were enrolled in a doubleblind, randomized 10-week trial consisting of 8 weeks of active drug with either weekly or daily administration of $50 \mathrm{mg}$ of DCS followed by a 2-week follow-up visit.

Results-For the purposes of this study, no statistical or clinical differences existed between the 2 dosage groups on the Aberrant Behavior Checklist subscale 3, which measures stereotypies/ repetitive movements. When combining groups, a statistically significant decrease of $37 \%$ was found from baseline to week 8 when study drug was completed using a linear mixed effects model $(P=0.003)$.

Conclusions-D-Cycloserine was shown to be effective in improving stereotypic symptoms in older adolescents and young adults with ASDs measured by the Aberrant Behavior Checklist subscale 3. In addition, DCS was safe and well tolerated.

\section{Keywords}

autism; social deficits; stereotypies; D-cycloserine 
Autism spectrum disorders (ASDs) comprise a group of neurodevelopmental disorders with core characteristic impairments in social communication as well as the presence of repetitive, stereotypic behaviors and restricted interests (RRBs). The prevalence of ASDs has dramatically increased with the current estimate of 1:88 children affected. ${ }^{1}$ Older adolescents and young adults (OAYA) with ASDs, who are particularly impacted by these deficits, remain a severely underserved population despite a recent surge in research and treatment services for patients with ASDs. ${ }^{2}$

Social communication deficits are especially difficult for this age population, whose focus is on independent living and separation from the family. ${ }^{2}$ For OAYA with ASDs, developmental tasks that include finding employment or educational training, establishing social networks with peers, attempting intimate relationships, and becoming self-sufficient in daily living skills do not occur smoothly if at all. Few specific treatments exist for the core social deficit in ASD. Psychotherapy trials have improved related behaviors that affect social function and/or anxiety. ${ }^{3}$ Medication trials alone or in conjunction with psychotherapies have improved the severity of behavioral symptoms and comorbid diagnoses, such as anxiety and depressive disorders. ${ }^{4-7}$ In addition, D-cycloserine (DCS), a glycine $_{B}$ partial agonist that acts at the N-methyl-D-aspartic acid (NMDA) receptor, was reported to have promising effects on sociability, a core symptom domain of ASDs. ${ }^{8}$ DCycloserine works cooperatively with glutamate to promote opening of the NMDA receptorassociated channel and $\mathrm{Ca}^{2+}$ conductance.

Repetitive, stereotypic behaviors and restricted interests can be further disabling and worsen the capacity to interact socially because they are bothersome to other individuals with whom the OAYA with an ASD is attempting to communicate. ${ }^{9,10}$ Repetitive, stereotypic behaviors and restricted interests include stereotyped motor mannerisms, restricted patterns of interest, atypical sensory interests, and the insistence for things to remain the same. ${ }^{11}$ Stereotypies appear involuntary in nature, have a pattern and rhythm that are predictable, are usually present in early childhood, and may diminish with age but usually remain present. ${ }^{11,12}$ Common behaviors include touching, sniffing, rubbing, hand flapping, picking, rocking, pacing, head banging, chewing movements, and facial grimacing. ${ }^{12}$ When compared with the number of studies focused on social communication, fewer have focused on specific research to address the RRB symptom domain. ${ }^{13}$ Behavioral interventions are mostly based on functional analysis and are consequence or antecedent-based. ${ }^{13}$ Psychopharmacologic trials have been mixed. ${ }^{11}$ Aripriprazole and risperidone, the 2 drugs approved by the Food and Drug Administration for treatment of ASDs, and haloperidol have shown some positive results when compared with placebo, whereas many other drugs such as atomoxetine, guanfacine, citalopram, and levetiracetam were partially effective or ineffective. ${ }^{14-16}$ Importantly, several of these studies used the Aberrant Behavior Checklist (ABC) subscale 3 (stereotypies) as the primary outcome measure. Despite the previously mentioned treatment trials, a gap continues to exist in the literature with regard to the effective treatment of stereotypies in OAYA with ASDs. ${ }^{11}$

Preclinical data implicate glutamatergic dysfunction in the pathophysiology of ASDs. ${ }^{4,17,18}$ For example, transgenic mice with deficient expression of the NR1 NMDA receptor subunit or an up to 5-fold diminished affinity of their NMDA receptors for the obligatory glycine 
co-agonist show evidence of impaired sociability ${ }^{19-21}$; specifically, these data support a regulatory role of the NMDA receptor in mouse sociability. Moreover, the genetically inbred Balb/c mouse strain, which serves as a mouse model of ASDs, has altered endogenous tone of NMDA receptor-mediated neurotransmission, as reflected in its heightened sensitivity to behavioral effects of MK-801 (dizocilpine), a noncompetitive NMDA receptor antagonist. Specifically, Balb/c mice are more sensitive to the elicitation of irregular episodes of intense jumping behavior, termed popping, and circling behavior, and antagonism of electrically precipitated seizures by MK-801 than many comparator mouse strains. ${ }^{22-24}$ Interestingly, the Balb/c mouse strain's deficits of sociability relative to the outbred Swiss Webster comparator strain, which include diminished locomotor activity in the presence of an enclosed or freely moving social stimulus mouse and fewer discrete episodes of social approach and anogenital sniffing of the stimulus mouse during their free interaction with each other, were improved by DCS. D-Cycloserine also improved sociability in the BTBR T+Itpr3tf/J (BTBR) mouse strain, another genetically inbred mouse model of ASDs, relative to the Swiss Webster comparator strain. ${ }^{25,26}$ Importantly, DCS significantly reduced grooming behavior in both BTBR and Swiss Webster strains, as well as rearing behavior in the Swiss Webster mouse ${ }^{25}$; grooming and rearing behaviors are examples of stereotypic, repetitive behaviors. The family of Shank scaffolding proteins and neuroligin 1, a postsynaptic adhesion molecule, is enriched in the postsynaptic compartment of excitatory synapses, where they are critical for the proper alignment and functioning of ionotropic and metabotropic glutamate receptors. Transgenic mice with deficient expression of Shank proteins and neuroligin 1 also serve as models of ASDs. ${ }^{21,27}$ Interestingly, DCS improved sociability and reduced the intensity of repetitive stereotyped grooming in some of these genetically engineered mice with deficient expression of Shank 2 and neuroligin 1, respectively. ${ }^{21}$ Collectively, these preclinical data implicate impaired glutamatergic neurotransmission in the pathogenesis of impaired sociability and RRBs. Moreover, the fact that the severity of impaired sociability and stereotypic behaviors are dissociated in Balb/c mice, relative to the Swiss Webster comparator strain, suggests that different circuit disturbances may underlie these 2 core symptom domains.

The sensitivity of the obligatory glycine ${ }_{\mathrm{B}}$ co-agonist binding site may change with daily administration of DCS as a result of "agonist-induced desensitization."28,29 Thus, in this pilot investigation, we compared the efficacy of a "pulsed" once-weekly administration versus "daily" administration of DCS to address the important issue of "tachyphylaxis" or receptor desensitization, which is a loss of efficacy due to tonic exposure to DCS as a result of daily administration. ${ }^{30}$ Importantly, a pulsed administration has been shown to be effective in terms of facilitating extinction in human subjects treated for obsessivecompulsive disorder with cognitive behavioral therapeutic interventions. ${ }^{5}$ Also, a "pulsed" administration has been studied in patients with schizophrenia, a disorder where DCS has been administered to target presumed NMDA receptor hypofunction. ${ }^{31}$

As noted previously, DCS was reported to improve sociability in children, adolescents, and young adults with ASDs. ${ }^{8}$ To the best of our knowledge, DCS has not been tested as a treatment for stereotypies in individuals with ASD. Because stereotypies aggravate social dysfunction in patients with ASDs, we wondered whether DCS would improve RRBs in 
OAYA with ASDs. ${ }^{9,10}$ Specifically, we evaluated the effect of DCS on RRBs, using the $\mathrm{ABC}$ subscale 3, in OAYA with ASDs in a trial comparing 2 dosage strategies: a pulsed, once-weekly dose of DCS (50 mg) versus a daily dose of DCS (50 mg). ${ }^{30}$ Importantly, a desired medication for the treatment of ASDs would improve sociability while not worsening stereotypies and vice versa.

\section{MATERIALS AND METHODS}

\section{Participants}

Males and females, ages 14 to 25 years, with a Diagnostic and Statistical Manual of Mental Disorders, Fourth Edition Text Revision documented diagnosis of an ASD from a psychologist/ psychiatrist and whose symptoms met a threshold score on the ASD checklist completed by a parent/primary caregiver were enrolled; the checklist is based on the Diagnostic and Statistical Manual of Mental Disorders, Fourth Edition Text Revision criteria and was developed by one of the co-authors (K.H.). The ASD checklist served to assure that groups were comparable with equivalent numbers of discrete symptoms but not necessarily similar with respect to symptom severity at the time of enrollment. All subjects were required to provide documentation of a normal physical examination, laboratory screen (comprehensive metabolic panel and complete blood count), electrocardiogram, and IQ test (IQ > 70) within a year before starting the study. Medication and therapy regimens were required to be stable for 4 weeks before the beginning of the study. Exclusion criteria included individuals with active suicidal thoughts, major medical or psychiatric diagnoses, pregnancy, breastfeeding, or taking isoniazids/ethionamides. While enrolled in the study, the participants agreed to maintain their stable treatment regimen and refrain from drinking alcohol for the duration of the study. All female participants were given a pregnancy test and excluded if the test yielded a positive result. Subjects with abnormal electrocardiogram results were referred to their cardiologist/ phencyclidine for medical clearance. All subjects or guardians (legally appointed representatives) provided voluntary informed consent or assent, as appropriate. This study was approved by the Eastern Virginia Medical School institutional review board. The location of the study was the Psychiatry and Behavioral Sciences, Eastern Virginia Medical School, Norfolk, VA.

\section{Design}

The study was a double-blind, randomized 10-week trial consisting of 8 weeks of active drug with either weekly or daily administration followed by a 2-week follow-up visit, conducted between August 2010 and March 2012. Two dosing strategies, $50 \mathrm{mg}$ weekly (placebo taken for the remaining 6 days of the week) and $50 \mathrm{mg}$ daily, were compared. The subjects were required to attend 4 visits: baseline, midpoint of active drug administration at 4 weeks, end of active drug administration at 8 weeks, and follow-up at 10 weeks. Study measures were completed at each visit. A diary card was completed on a daily basis for 10 weeks to assess for adverse effects.

\section{Study Measures}

Study measures were administered at each visit. The primary study measure, the Social Responsiveness Scale (SRS), measures the severity of autism in an individual's natural 
setting and focuses on social awareness, social information processing, capacity for reciprocal social communication, social anxiety/ avoidance, and autism preoccupation. ${ }^{32}$ Secondary measures included the ABC, which is a measure used to assess drug and other treatment effects (psychopharmacologic, behavioral, and others) in intellectually disabled individuals and persons with ASDs. ${ }^{33,34}$ The $\mathrm{ABC}$ is normed from childhood to adulthood and has 58 items rated on a Likert scale. Factor analyses of the $\mathrm{ABC}$ resulted in 5-factor subscales: (1) Irritability, Agitation, Crying; (2) Lethargy, Social Withdrawal; (3) Stereotypic Behavior; (4) Hyperactivity, Noncompliance; and (5) Inappropriate Speech. ${ }^{33,34}$ The effect of DCS on the SRS and ABC subscale 2 was reported elsewhere. ${ }^{30}$ The ABC subscale 3 or the stereotypy subscale is the focus of this publication; it is a 7-item subscale that focuses on repetitive stereotypic movements (eg, rocking, hand flapping, repetitive movements, and odd bizarre motor behaviors).

\section{Statistical Analysis}

Summary statistics included mean and standard deviation for continuous variables and frequencies for categorical variables. Linear mixed effects models were used to assess changes in clinical outcomes over time according to group assignment by testing for the additive effects of group and time as well as group-time interaction. $t$ Tests were used to test for differences in baseline demographics between the 2 groups. A significance level of $a=$ 0.05 was used for all analyses. $P$ values were adjusted for multiple comparisons using the Bonferroni correction. SAS version 9.2 was used for all analyses.

\section{RESULTS}

A total of 21 subjects were enrolled in the study and randomized to either daily or weekly dosage of DCS using a block randomization procedure. Differences in demographics between the 2 groups were insignificant and are reported elsewhere. ${ }^{30}$ One subject discontinued DCS after the baseline visit because of minor depression, resulting in a total of 20 subjects in trial. Importantly, DCS was very well tolerated; only transient spontaneously recorded adverse effects were noted on "diary cards" kept by the patients and their caregivers, which were not reasons for medication discontinuation. ${ }^{30}$ All subjects maintained a stable medication and therapy regimen throughout the trial. Most subjects were taking a serotonin enhancing drug (eg, selective serotonin reuptake inhibitors, buspirone, clomipramine $[\mathrm{n}=13])$ and/or a stimulant (eg, methylphenidate extended release, methylphenidate, atomoxetine, dextroamphetamine/amphetamine $[n=9])$. Three subjects were taking low-dose risperidone or aripiprazole; 1 subject, clonidine; and 1 subject, oxcarbazepine.

For the purposes of this study, no statistical or clinical differences existed between the 2 dosage groups on the SRS, the primary outcome measure of efficacy. ${ }^{30}$ The secondary outcome measures of the ABC subscales showed no clinical or statistical differences between the dosage groups on subscale 2 (social/lethargy) and subscale 3 (stereotypies). Importantly, when all subscales of the $\mathrm{ABC}$ were analyzed on the combined or individual dosage strategies, $\mathrm{ABC}$ subscale 3, which measures stereotypies/repetitive movements, was found to have a statistically significant decrease from baseline to week 8 when study drug 
was completed by a linear mixed effects model $(P=0.003)$. When combining groups, there was a $37 \%$ decrease in mean $\mathrm{ABC}$ subscale 3 score from baseline to week 8 of 1.6 points. A graph of the combined results of the $\mathrm{ABC}$ subscale 3 is pictured in Figure 1.

\section{DISCUSSION}

Autism spectrum disorders are life-long disabling conditions that affect many realms of a patient's life. Both social impairment and repetitive behaviors are especially problematic for the OAYA group, who are attempting significant developmental life changes. Although progress has been made in finding both psychotherapeutic and psychopharmacologic treatments for ASD, few, if any, target the core symptom domains specifically and include the OAYA population with ASDs.

D-Cycloserine was shown to be effective in improving stereotypic symptoms in OAYA with ASDs on the ABC subscale 3; in addition, DCS was safe and well tolerated. Total scores at baseline were low, with half of the subjects having a score of 3 or less of 21 possible points, so a floor effect was noted; this may reflect the fact that selection criteria included subjects with normal or near-normal full-scale IQ (IQ, >70) and good expressive language skills. ${ }^{30}$ Thus, it is possible that subjects with more severe stereotypic symptoms would show clinically significant, in addition to statistically significant, improvement after taking DCS. Further, whereas $\mathrm{ABC}$ subscale 3 focuses specifically on movement disorders, it would be interesting to explore effects of DCS on other RRB criteria (eg, the need for sameness, restricted interests, or rigidity of schedule). Other limitations of this study include a lack of placebo control although the subjects were randomized and both raters and subjects were blinded to dosing condition. Moreover, a small sample size with high variability on the outcome measures limits the conclusion that there is no difference between dosing strategies. A larger sample size may augment the data pertaining to the clinical significance of DCS in treating patients with ASDs, and additional measures of RRBs may confirm this treatment effect. Bias from parental reporting is also a consideration. Finally, the combination of DCS with a serotonin-enhancing drug may be responsible for the positive results because several of our responders were taking one of these medications.

In any event, DCS, a well-tolerated drug with few adverse effects, is shown here to produce statistically significant improvement in both social deficits and RRBs and clinically significant improvement in social deficits, the major impairments in ASDs. Future studies should focus on replicating and expanding these results as both a primary treatment or as an adjunct to other psychotherapeutic and/or psychopharmacologic treatments.

\section{Acknowledgments}

Conflicts of Interest and Source of Funding: This work was supported by the Hampton Roads Community Foundation (grant 20101482). The funding source had no involvement in the research. Bayview Pharmacy provided the study drug, and Eastern Virginia Medical School Psychiatry and Behavioral Sciences provided funding for study drug, staff, and supplies. 


\section{REFERENCES}

1. [December 20, 2013] Prevalence of Autism Spectrum Disorders-Autism and Developmental Disabilities Monitoring Network, 14 Sites, United States. 2008. Available at: http://www.cdc.gov/ mmwr/preview/mmwrhtml/ss6103a1.htm?s_cid=ss6103a1_w.

2. Wehman, P.; Smith, M.; Schall, C. Autism and the Transition to Adulthood: Success Beyond the Classroom. Paul H. Brookes; Baltimore, MD: 2009.

3. Reichow B, Steiner AM, Volkmar F. Cochrane review: social skills groups for people aged 6 to 21 with autism spectrum disorders (ASD). Evid-Based Child Heal Cochrane Rev J. 2013; 8(2):266315.

4. Posey DJ, Erickson CA, McDougle CJ. Developing drugs for core social and communication impairment in autism. Child Adolesc Psychiatr Clin N Am. 2008; 17(4):787-801. [PubMed: 18775370]

5. Wilhelm S, Buhlmann U, Tolin DF, et al. Augmentation of behavior therapy with D-cycloserine for obsessive-compulsive disorder. Am J Psychiatry. 2008; 165(3):335-341. [PubMed: 18245177]

6. Heaton LJ, McNeil DW, Milgrom P. Propranolol and D-cycloserine as adjunctive medications in reducing dental fear in sedation practice. SAAD Dig. 2010; 26:27-35. [PubMed: 20151608]

7. Rothbaum BO. Critical parameters for D-cycloserine enhancement of cognitive-behaviorial therapy for obsessive-compulsive disorder. Am J Psychiatry. 2008; 165(3):293-296. [PubMed: 18316423]

8. Posey DJ, Kem DL, Swiezy NB, et al. A pilot study of D-cycloserine in subjects with autistic disorder. Am J Psychiatry. 2004; 161(11):2115-2117. [PubMed: 15514414]

9. Campbell M, Locascio JJ, Choroco MC, et al. Stereotypies and tardive dyskinesia: abnormal movements in autistic children. Psychopharmacol Bull. 1990; 26(2):260-266. [PubMed: 2236468]

10. Goldman S, Wang C, Salgado MW, et al. Motor stereotypies in children with autism and other developmental disorders. Dev Med Child Neurol. 2009; 51(1):30-38. [PubMed: 19087102]

11. Carrasco M, Volkmar FR, Bloch MH. Pharmacologic treatment of repetitive behaviors in autism spectrum disorders: evidence of publication bias. Pediatrics. 2012; 129(5):e1301-e1310. [PubMed: 22529279]

12. Barry S, Baird G, Lascelles K, et al. Neurodevelopmental movement disorders-an update on childhood motor stereotypies. Dev Med Child Neurol. 2011; 53(11):979-985. [PubMed: 21749367]

13. Boyd BA, McDonough SG, Bodfish JW. Evidence-based behavioral interventions for repetitive behaviors in autism. J Autism Dev Disord. 2012; 42(6):1236-1248. [PubMed: 21584849]

14. Asadabadi M, Mohammadi M-R, Ghanizadeh A, et al. Celecoxib as adjunctive treatment to risperidone in children with autistic disorder: a randomized, double-blind, placebo-controlled trial. Psychopharmacology (Berl). 2013; 225(1):51-59. [PubMed: 22782459]

15. Hardan AY, Fung LK, Libove RA, et al. A randomized controlled pilot trial of oral Nacetylcysteine in children with autism. Biol Psychiatry. 2012; 71(11):956-961. [PubMed: 22342106]

16. Rajapakse T, Pringsheim T. Pharmacotherapeutics of Tourette syndrome and stereotypies in autism. Semin Pediatr Neurol. 2010; 17(4):254-260. [PubMed: 21183132]

17. Marino MJ, Conn PJ. Glutamate-based therapeutic approaches: allosteric modulators of metabotropic glutamate receptors. Curr Opin Pharmacol. 2006; 6(1):98-102. [PubMed: 16368268]

18. Javitt DC. Glutamate as a therapeutic target in psychiatric disorders. Mol Psychiatry. 2004; 9(11): 984-997. [PubMed: 15278097]

19. Halene TB, Ehrlichman RS, Liang Y, et al. Assessment of NMDA receptor NR1 subunit hypofunction in mice as a model for schizophrenia. Genes Brain Behav. 2009; 8(7):661-675. [PubMed: 19563516]

20. Labrie V, Lipina T, Roder JC. Mice with reduced NMDA receptor glycine affinity model some of the negative and cognitive symptoms of schizophrenia. Psychopharmacology (Berl). 2008; 200(2): 217-230. [PubMed: 18597079]

21. Won H, Lee H-R, Gee HY, et al. Autistic-like social behaviour in Shank 2-mutant mice improved by restoring NMDA receptor function. Nature. 2012; 486(7402):261-265. [PubMed: 22699620] 
22. Deutsch SI, Rosse RB, Paul SM, et al. Inbred mouse strains differ in sensitivity to "popping" behavior elicited by MK-801. Pharmacol Biochem Behav. 1997; 57(1-2):315-317. [PubMed: 9164588]

23. Deutsch SI, Mastropaolo J, Powell DG, et al. Inbred mouse strains differ in their sensitivity to an antiseizure effect of MK-801. Clin Neuropharmacol. 1998; 21(4):255-257. [PubMed: 9704168]

24. Burket JA, Cannon WR, Jacome LF, et al. MK-801, a noncompetitive NMDA receptor antagonist, elicits circling behavior in the genetically inbred Balb/c mouse strain. Brain Res Bull. 2010; 83(6): 337-339. [PubMed: 20813169]

25. Burket JA, Benson AD, Tang AH, et al. D-Cycloserine improves sociability in the BTBR T+ Itpr3tf/J mouse model of autism spectrum disorders with altered Ras/Raf/ERK1/2 signaling. Brain Res Bull. 2013; 96:62-70. [PubMed: 23685206]

26. Deutsch SI, Pepe GJ, Burket JA, et al. D-cycloserine improves sociability and spontaneous stereotypic behaviors in 4-week old mice. Brain Res. 2012; 1439:96-107. [PubMed: 22261249]

27. Blundell J, Blaiss CA, Etherton MR, et al. Neuroligin-1 deletion results in impaired spatial memory and increased repetitive behavior. J Neurosci. 2010; 30(6):2115-2129. [PubMed: 20147539]

28. Goff DC, Cather C, Gottlieb JD, et al. Once-weekly D-cycloserine effects on negative symptoms and cognition in schizophrenia: an exploratory study. Schizophr Res. 2008; 106(2-3):320-327. [PubMed: 18799288]

29. Quartermain D, Mower J, Rafferty MF, et al. Acute but not chronic activation of the NMDAcoupled glycine receptor with D-cycloserine facilitates learning and retention. Eur J Pharmacol. 1994; 257(1-2):7-12. [PubMed: 8082709]

30. Urbano MR, Okwara L, Manser P, et al. A trial of D-Cycloserine to treat the social deficit in older adolescents and young adults with autism spectrum disorders. J Neuropsychiatry Clin Neurosci. In press.

31. Goff DC. D-cycloserine: an evolving role in learning and neuroplasticity in schizophrenia. Schizophr Bull. 2012; 38(5):936-941. [PubMed: 22368237]

32. Constantino, J.; Gruber, C. The Social Responsiveness Scale (SRS) Manual. Western Psychological Services; Los Angeles, CA: 2005.

33. Aman MG, Kasper W, Manos G, et al. Line-item analysis of the Aberrant Behavior Checklist: results from two studies of aripiprazole in the treatment of irritability associated with autistic disorder. J Child Adolesc Psychopharmacol. 2010; 20(5):415-422. [PubMed: 20973712]

34. Aman MG, Singh NN, Stewart AW, et al. The aberrant behavior checklist: a behavior rating scale for the assessment of treatment effects. Am J Ment Defic. 1985; 89(5):485-491. [PubMed: 3993694] 


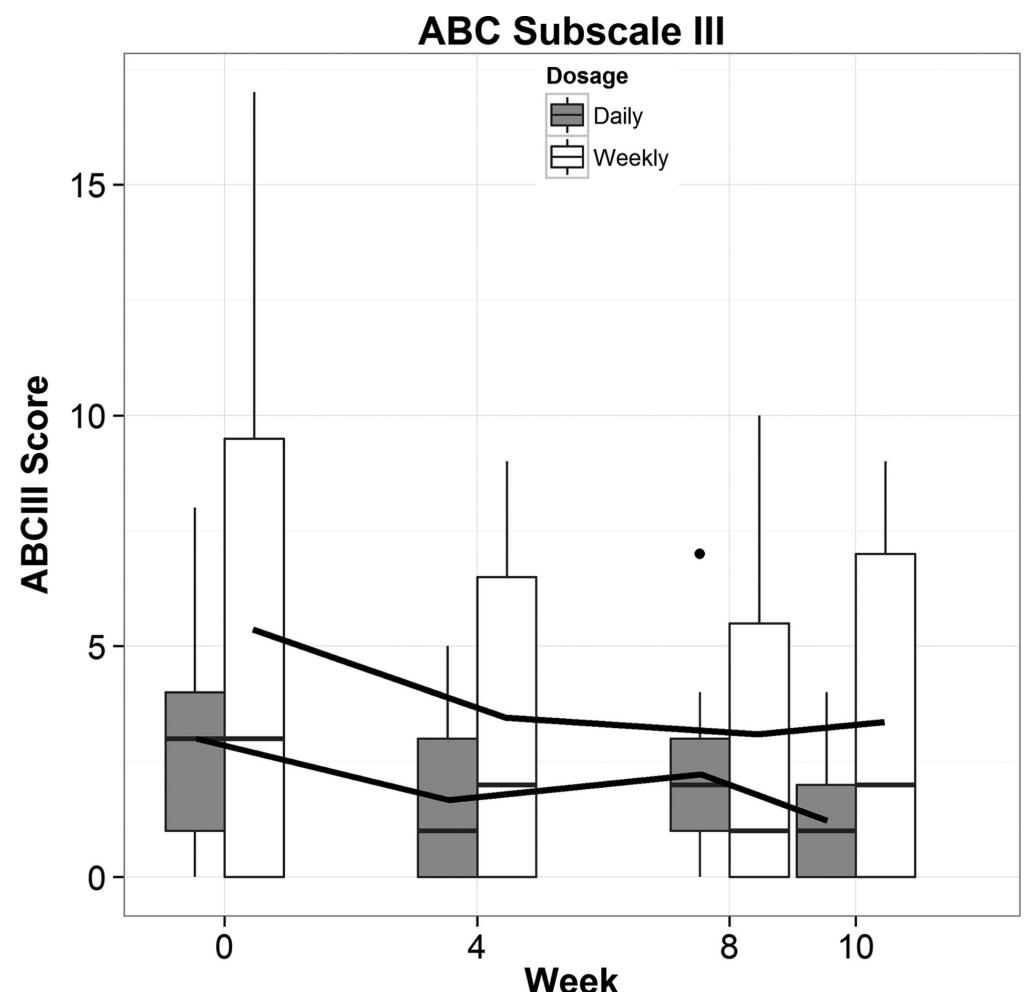

FIGURE 1.

Stereotypy deficit response to DCS (ABC III) No statistical or clinical differences were seen between the dosage groups (group-time interaction). Both daily and weekly dosing strategies showed a significant downward linear trend $(P=0.003)$ with a $37 \%$ decrease in mean ABC subscale 3 score from baseline to week 8 of 1.6 points. Total scores at baseline were low with half of the subjects having a score of 3 or less of 21 possible points, so a floor effect was noted. 